\title{
Proposals of Cogeneration System Alternatives as Cost Reduction Factor in the Use and Consumption of Energy and Pollutant Emission Gases in Alpha Hospital in Manaus City
}

\author{
Victor da Silva Almeida', Jorge Laureano Moya Rodriguez², Maida Bárbara Reyes Rodríguez², \\ Jandecy Cabral Leite ${ }^{2}$
}

${ }^{1}$ Programa de Pós-Graduação em Engenharia de Processos do Instituto de Tecnologia da Universidade Federal do Pará (ITEC-UFPA). Rua Augusto Correa, 01 - Guamá. CEP: 66075-110. Caixa Postal 479. PABX + 5591 3201-7000. Belém Pará - Brasil.

${ }^{2}$ Instituto de Tecnologia e Educação Galileo da Amazônia (ITEGAM). Avenida Joaquim Nabuco No 1950. Centro. CEP: 69005-080. Manaus - Amazonas - Brasil.

Email: admvictor1@gmail.com ,jorgemoyar@gmail.com, maidabrr@gmail.com,jandecy.cabral@itegam.org.br

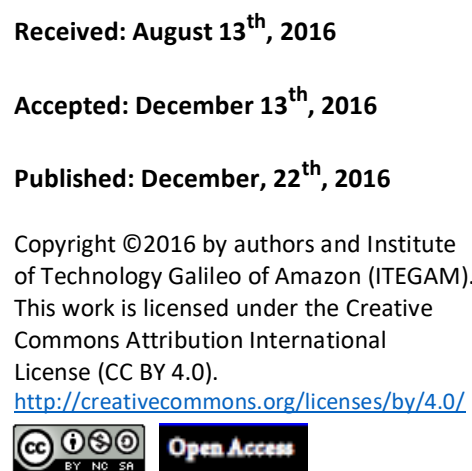

\begin{abstract}
Hospitals have the highest energy consumption in units per area in the buildings. Continuous use of heating and cooling equipment requires greater attention in all directions in order to maintain satisfactory thermal comfort and indoor air quality levels for patients. Knowing that this structure has a cost with electric energy consumption, this work was developed from the structural analysis of the Alpha hospital, where it was surveyed its attendance capacity, equipment that requires the use of electric energy and the consume periods, considering the climate. From this, it was analyzed the equipment that is needed to implement a cogeneration module in the hospital, proposing two possibilities and analyzing its economic viability and return on investment in the medium and long term.
\end{abstract}

Keywords: Cogeneration, cost reduction, competiveness, environmental impact.

Propostas Alternativas de Sistema de Cogeração como fator de Redução de Custo no Uso e Consumo de Energia Elétrica e Emissão de Gases Poluentes no Hospital Alpha da cidade de Manaus.

\section{RESUMO}

Hospitais têm o maior consumo de energia por área nas unidades dos setores dos edifícios. A utilização contínua de equipamento de aquecimento e de resfriamento requer uma atenção maior em todos os sentidos, de modo a manter o conforto térmico satisfatório e níveis de qualidade do ar interior para os pacientes. E sabendo que esta estrutura tem um custo com consumo de energia elétrica, esse trabalho foi desenvolvido a partir da análise estrutural do hospital Alpha, onde foi feito levantamento da capacidade de atendimento do mesmo, equipamentos que requerem o uso de energia elétrica e os períodos de consumos, considerando o clima inclusive. A partir disso, foi analisado os equipamentos que são necessários para implantação de um modulo de cogeração no hospital, propondo-se três possibilidades e analisando sua viabilidade econômica e o retorno sobre investimento a médio e longo prazo.

Palavras Chaves: Cogeração, Redução de Custo, Competitividade, Impacto Ambiental. 


\section{INTRODUÇÃO}

Em função do crescimento populacional brasileiro, o sistema elétrico nacional está cada vez mais em modificação a fim de atender as necessidades do país. Na última década, ganhou destaque o aumento da geração termoelétrica na matriz elétrica nacional, primeiro proveniente de gás natural, e mais recentemente, de fontes renováveis de energia como a geração proveniente de biomassa ou térmica útil. Isto por que, a partir da década de 1970, com a ocorrência de duas crises internacionais envolvendo o abastecimento de petróleo, a primeira em 1973 e a segunda em 1979, é que o mundo tomou consciência de que os recursos energéticos são finitos [1].

Em diversos países, com exceção dos Estados Unidos e Brasil, ao primeiro sinal de elevação exagerada dos preços do petróleo, programas de racionalização do uso da energia começaram a ser elaborados. Intensificando-se a discussão acerca do problema da utilização de energia, no qual o Brasil iniciou programas de incentivo à redução da demanda de energia elétrica primária contribuindo assim com a necessidade de se pensar propostas alternativas de geração de energia [1]

Desta forma, num sentido amplo, a conservação de energia engloba não apenas a diminuição da quantidade primária, necessária para propiciar o consumo de um mesmo nível útil, mas também a construção de um estilo alternativo de desenvolvimento que implique mais baixo perfil de demanda de energia útil para um mesmo padrão de satisfação das necessidades sociais. E nessa linha de raciocínio [1] diz que "entende-se por estilo de desenvolvimento a maneira como se organizam os recursos materiais e humanos dentro de um determinado sistema, com o objetivo de resolver as questões relativas à "o que", "para quem" e "como" produzir os bens e serviços". Analisando, então este contexto sabe-se que o passado histórico do país, especialmente sua relação com os demais países, assim como a capacidade de resolver seus próprios problemas, tem buscado soluções originais para problemas cada vez mais complexos.

Neste cenário se incorpora a ideia de cogeração, que segundo [2] trata-se de um sistema de produção de energia de alto rendimento, que consiste na produção simultânea de energia térmica e de energia eléctrica a partir de uma energia primária combustível, como as energias eletromecânica e térmica, para suprir as necessidades de uma unidade de processo, seja ela do setor industrial, hospitalar, agrícola, terciário ou um sistema isolado, a partir de uma mesma fonte energética primária. Em outras palavras, seria o aproveitamento de uma parcela de energia que teria de ser obrigatoriamente rejeitada por força da Segunda Lei da Termodinâmica, pois tende a incrementar-se com o tempo, até alcançar um valor máximo. Mais sensivelmente, quando uma parte de um sistema fechado interage com outra parte, a energia tende a dividir-se por igual, até que o sistema alcance um equilíbrio térmico, resultando em um aumento da eficiência energética global do ciclo térmico.

Para [3] analisam do ponto de vista da Cogeração, pressupondo-se que as questões energéticas que se envolvem nesse procedimento tendem a seguir o mesmo raciocínio lógico quando da análise das faturas energéticas, cujos custos marginais de expansão projetam valores a curto, médio e longo prazo com investimentos em equipamentos e máquinas que irão reduzir substancialmente o gasto com energia elétrica.

Ratificando a característica estratégica dessa modalidade alternativa de produção simultânea de energia a partir da térmica útil com a convencional, demonstra-se que devido às elevadas taxas de cobrança das redes de energias atuais, bem como a utilização em empresas do segmento hospitalar em grandes volumes devido à natureza da atividade, requer-se um novo modelo de se utilizar e pensar o consumo energético sustentável. Conforme se verifica a necessidade de eficiência energética em edifícios hospitalares em que se demonstra que a Cogeração traz benefícios diversos como ampliação da potência de frio, centralização da produção de energia dentre outros [4].

Confirmando esse contexto [5] mostra que o Sistema de cogeração (CHP) é uma das maneiras de economizar a energia e utilizar a energia eficientemente. Quando comparadas para separar a geração de combustível fóssil do calor e eletricidade, CHP pode resultar numa poupança de energia consistente (normalmente variando de $10 \%$ a $30 \%$ ), enquanto que as emissões de $\mathrm{CO}_{2}$ são evitadas, como uma primeira aproximação, semelhante ao valor de energia poupada. E em termos de sustentabilidade, uma das principais considerações é a eficiência da energia, pois a energia sustentável é considerada como um tipo de energia que é renovável e contínua, o que significa que o uso desse tipo de energia pode, potencialmente, ser mantido no futuro sem causar repercussões negativas para as gerações futuras.

\section{REVISÃO BIBLIOGRÁFICA}

A Cogeração teve sua origem na Europa e nos Estados Unidos ao final do século XIX. Durante as primeiras décadas do século XX, a maioria das indústrias tinham suas próprias unidades de geração de potencia com sistemas de caldeira de vapor e turbina funcionando com carbono e muitas dessas unidades de cogeração. Posteriormente lhe seguiu um período no qual a aplicação decresceu.

E por dar uma ideia de magnitude acerca do que se tem falado, no inicio do século XX se gerava, pela cogeração, mais da metade da energia elétrica em EEUU, fazendo em 1950 esse valor cair de uns $15 \%$ a uns $5 \%$ na década de 70 . O desenvolvimento de grandes plantas de potencia que proporcionavam um fornecimento confiável de eletricidade e a alta disponibilidade de petróleo, além do barateamento dos combustíveis para as centrais térmicas foram as duas causas principais que fizeram com que as inversões nas instalações de cogeração fossem pouco rentáveis, exceto nos casos onde as indústrias tinham calor residual dos seus processos e estiveram ligadas a rede elétrica.

Posteriormente, a crise energética de 1973 provocou uma grande preocupação a nível industrial, que originou no estabelecimento de normativas (National) Energy Act em 1973 e publicou Utility Regulatory Policy, PURPA em 1978 para assegurar o fornecimento energético, estabelecendo os princípios básicos para a compra da eletricidade produzida pelos cogeradores e pelas plantas elétricas de pequena potência. Com isto, se originou o inicio do cenário mudando de um número cada vez maior de iniciativas e projetos de cogeração [6]. 
Mais recentemente, em 1993, o Comitê Internacional da Energia da OECD (Organization for Economic Cooperation and Development) na intenção de criar condições nas quais o setor energético fora capaz de contribuir para o desenvolvimento, estabeleceram vários objetivos, entre eles o de diversificar, fazer mais eficiente e flexibilizar o setor energético, desenvolvendo fontes de energia ambientalmente aceitáveis e um mercado mais competitivo. Isto contribuiu com a política que a Europa implantava delineado em três objetivos principais em matéria energética: A segurança de abastecimento, combate ao aquecimento global e competitividade, identificando a cogeração como uma das ferramentas para alcançar estes três objetivos [7].

Atualmente, os governos como os da Europa, E.U.A, Canadá e Japão, principalmente, estão promovendo o uso da cogeração somente no setor industrial. Na Europa, por meio da diretriz 2004/08/CE, se planeja o fomento da cogeração, onde o objetivo principal é incrementar a eficiência energética e melhorar a segurança do abastecimento mediante a criação de um marco para o fomento e desenvolvimento da Cogeração de alta eficiência. A diretriz se estabelece de que para atingir o objetivo deve-se ter em conta as circunstâncias nacionais e específicas. Assim, no caso da Espanha em 2006 se contava com 6.500 MWe (15\% da demanda elétrica) instalados em plantas de cogeração e se espera contar com 8.400 WME até 2012, cobrindo os $20 \%$ da energia elétrica consumida [7].

\section{II.1 SISTEMAS DE COGERAÇÃO}

A seleção de um sistema que garanta o fornecimento adequado de energia a uma instalação é um processo que compreende entre outros aspectos a concordância das demandas do tempo, ou o que eles mesmos e a simultaneidade das demandas. Por outro lado se encontram os custos das diferentes tecnologias, os preços de compra e venda, as políticas regulatórias e as estratégias de operação. Este processo tendo em conta a diversidade de sistemas e possibilidades combinações se faz complexo ao analisa-lo segundo os métodos tradicionais. Na atualidade se empregam ferramentas computacionais para chegar ao melhor tempo possível no modelo adequado de combinação de equipamentos, tendo em vista o alcance da máxima eficiência e o menor custo.

Para [8] diz que atualmente existem diferentes sistemas de informação disponíveis a nível acadêmico e profissional. Onde estes sistemas são muito úteis para sensibilizar o usuário com as ordens de magnitude e as possíveis combinações de tecnologia, porém raramente proporcionam um resultado acordado em cada situação particular.

Para chegar a um planejamento que considere a síntese, o desenho e a operação de forma conjunta dos sistemas de cogeração, deve-se empregar procedimentos de otimização de onde se busca encontrar a configuração da planta, dimensionando seus equipamentos e avaliar a melhor estratégia de operação. No modelo se podem adicionar certas restrições para valorizar o benefício econômico, a economia de energia e o impacto ambiental [8].

O processo de otimização de pode distinguir em várias etapas, porém, basicamente se pode identificar dois: Um nível de procedimento de projetos e um nível de avaliação de comportamento baixo da influência das variáveis. Sendo, no procedimento do projeto se busca otimizar o tamanho dos dispositivos que confirmam a planta e os parâmetros de configuração, enquanto que no procedimento de avaliação de comportamento, se busca identificar a melhor estratégia de operação [9].

\section{MATERIAIS E MÉTODOS}

A metodologia é definida como procedimentos, conjunto de abordagens, técnicas e processos utilizados pela ciência para formular e resolver problemas de aquisição objetiva do conhecimento, de uma maneira sistemática, envolvendo a apresentação de informações de alguns aspectos como: tipo de pesquisa, instrumentos utilizados (questionário, entrevista etc), população e amostra, coleta dos dados e análise dos dados [10].

Este trabalho se caracteriza como um estudo exploratório, pois [11] afirmam que "a pesquisa exploratória é caracterizada pela existência de ideias, problemas e insights, sendo flexível e versátil, além de se poderem utilizar dados secundários e pesquisas qualitativas". E confirmando nesse contexto em que nasce o estudo, será descrito, um modelo de Cogeração adequado para um hospital, que embora a implementação deste modelo tenha como um objetivo abordar em algum trabalho posterior no presente, um modelo de programação e otimização de processos, este modelo que foi definido a partir dos diversos modelos existentes, escolhendo-se para implementação o modelo físico por componente, porquê mostra a configuração com motor de combustão interna (MACI) ou turbina a gás, destacando seu consumo de combustível (Fuel) para produzir eletricidade (PGen) e aproveita os gases de escape para produzir vapor (HRec), sendo este, um dos fatores de análise da proposta estudada, gerando indicadores inclusive dos resultados das variantes de consumo de energia elétrica.

\section{III.1 PROJETO DE PESQUISA}

Com a finalidade de se atingirem os objetivos almejados neste estudo, foram necessários realizar várias etapas para o desenvolvimento, sendo que primeiramente procurou-se averiguar na literatura, uma fundamentação teórica sobre Cogeração e o modelo para diagnosticar, formular, implantar e verificar os resultados da implantação e o seu TIR (Taxa Interna de Retorno). Na segunda etapa, foi escolhida a empresa a ser implantado o programa, onde deu início com definição do hospital o qual denominamos nesse estudo como sendo ALPHA, pois o nome oficial não será revelado, sob o argumento de sigilo e também sentia a necessidade de melhoria na empresa, visto que possui um objetivo de se tornar referência no segmento e também como responsabilidade, pois faz seus serviços sob uso consciente de recursos. Foi definido que a análise estrutural seria feita em todo o hospital, verificando os equipamentos que o mesmo possui, leitos, layout e como os mecanismos hidráulicos e demais instalações estão distribuídos, para assim chegar nos resultados esperados.

$\mathrm{Na}$ Terceira etapa optou-se pela escolha de um modelo físico de cogeração a ser implementado, uma vez que se fez observação da estrutura do hospital e como se da o consumo de 
combustível no âmbito do mesmo em quatro categorias, a saber: Energia elétrica, Gás Natural, Fuel Oil e Diesel. A fim de fazer uma comparação entre as variáveis de consumo. No hospital se consomem quantidades apreciáveis de energia elétrica e esse consumo se utiliza a tonelada de combustível convencional (tcc) como medida padrão (usando os fatores de conversão).

$\mathrm{Na}$ quarta etapa, são apresentadas propostas alternativas de implantação de sistemas de cogeração e cada um com sua característica, estrutura de equipamentos necessários para implementação e o valor do investimento e seu retorno a médio prazo, tendo em vista o objetivo do trabalho quanto a redução de custo do consumo energético convencional e redução de emissão de gases poluentes atmosféricos. Já na quinta e última etapa, foi feita a análise dos dados, sendo o objetivo do tratamento e análise de dados descrever, interpretar, categorizar e explicar os dados coletados, de maneira que esses venham a responder as questões formuladas no estudo.

\section{III.2 DESCRIÇÃO DO CASO - HOSPITAL ALPHA}

O Hospital Clínico Cirúrgico Alpha é uma instituição do Sistema Nacional de Saúde que iniciou suas atividades em 20 de novembro de 1980. Este hospital se encarrega de ofertar assistência médica, científica e tecnológica altamente qualificada, não só no seu local de atuação, mas em todo o território nacional, pois está equipado com tecnologias de ponta e conta com um grupo importante de especialistas reconhecidos nacionalmente.

Nele são prestados serviços hospitalares nas três áreas básicas: internação, hospitalização e consulta externa. Atende aproximadamente uma população de 600 mil habitantes. O hospital conta com 20 salões de operações e uns 700 leitos disponíveis, neste momento tem se trabalhado par abrir quatro novas salas de serviços hospitalares como: hemodiálise, angiologia, urologia e geriatria, com o objetivo de aumentar a capacidade e qualidade dos serviços prestados.

No hospital Alpha se consomem quantidades apreciáveis de energia elétrica, Fuel Oil, GLP e Diesel, e os altos consumos de energia elétrica do hospital se devem ao grande número de equipamentos elétricos médicos e de serviços que existem no mesmo, estes na maioria dos casos são altos consumidores de eletricidade e muitos deles tem um fator de utilização elevado devido a função que desempenham e em sua maioria não podem ser desconectados. O hospital está ainda, equipado com um número elevado de tecnologias de ponta, porém, nele também coexistem tecnologias ultrapassadas e em mal estado técnico que faz do mesmo um centro ineficiente dentro do ponto de vista energético. Para a aplicação das ferramentas da TGTEE no hospital se selecionaram o período desde o ano de 2008 até 2012, neste período se instalaram novos equipamentos de serviços médicos. E a partir dos dados estatísticos coletados na economia e no próprio departamento estatístico para os anos analisados, se identificaram os principais consumidores energéticos do período: Energia Elétrica; Fuel Oil GLP e Diesel.

Se destaca que todos os tipos empregados são do tipo não renováveis, constatou-se que na entidade não se empregam fontes renováveis de energia.

Energia elétrica - Se monitorou o consumo de energia elétrica e outras magnitudes relacionadas nos horários de madrugada, dia de pico, assim como de reação para os anos 2008 à 2012 excluindo o ano de 2011, pois não se pôde coletar a informação necessária por falta de documentação na entidade.

Petróleo Combustível (FUEL OIL) - A segunda em importância fonte primária de energia consumida no hospital é o petróleo combustível que serve essencialmente para a produção de vapor na planta de geração de vapor. Esta conta com duas caldeiras piro-tubulares em funcionamento e uma que está sendo instalada e que produz vapor como fonte intermediária de energia e que serve as demandas de calor das diferentes dependências que o requerem, a saber: Lavanderia, Cozinha, Esterilização e Aquecimento de água. Pela quebra dos aquecedores de agua, atualmente não se presta este último serviço, foi coletada a informação disponível sobre as partículas de insumo de petróleo combustível durante o ano de 2012.

\section{RESULTADOS}

A análise econômica das diferentes alternativas se baseia em uma análise de pré-viabilidade das variáveis que energeticamente são idôneas, para assim tomar uma alternativa mais realista economicamente.

\section{IV.1 FORNECEDORES DE EQUIPAMENTOS, CUSTOS DE INVESTIMENTO E MANUTENÇÃO.}

Para realizar o cálculo de pré-viabilidade é necessário ter uma relação dos possíveis equipamentos a utilizar, dos mesmos é necessário conhecer suas capacidades, seus custos, etc. A tabela 1 apresenta um resumo das principais tecnologias de cogeração que são interesse para esse trabalho, na mesma aparecem dados da eficiência e o custo das tecnologias energéticas em exploração e desenvolvimento, da atualidade.

Tabela 1. Características Gerais e Custos de Algumas Tecnologias de Cogeração.

\begin{tabular}{l|c|c|c|c|c}
\hline \multicolumn{1}{c|}{ Tecnologia } & Potência [MW] & $\begin{array}{c}\text { Eficiência } \\
\text { Elétrica (\%) }\end{array}$ & $\begin{array}{c}\text { Eficiência Global } \\
(\mathbf{\%})\end{array}$ & $\begin{array}{c}\text { Investimento } \\
\text { (USD/kW) }\end{array}$ & $\begin{array}{c}\text { Custo O\&M } \\
\text { (USD/kWh) }\end{array}$ \\
\hline Turbina de Vapor & $0,25-500$ & $12-25$ & $60-80$ & $200-1.800$ & 0,0027 \\
\hline Turbina de Gás & $0,25-50$ & $25-42$ & $65-87$ & $400-8.500$ & $0,004-0,009$ \\
\hline Microturbina de Gás & $0,005-0,1$ & $15-30$ & $60-85$ & $600-850$ & $0,006-0,01$ \\
\hline $\begin{array}{l}\text { MACI (otto e } \\
\text { Diesel) }\end{array}$ & $0,003-20$ & $25-45$ & $65-92$ & $300-1.450$ & $0,007-0,014$ \\
\hline
\end{tabular}

Fonte: Autores, (2016).

$\mathrm{Na}$ Tabela 2 aparecem alguns fornecedores de equipamentos para condicionamento de ar que trabalham com 
ciclo de absorção, estes são os mais comercializados atualmente.

No citado quadro a primeira marca e a última que aparecem são de

fabricação chinesa e o segundo de fabricante alemã.

Tabela 2. Características e Custos de alguns equipamentos de refrigeração por absorção.

\begin{tabular}{c|c|c|c|c}
\hline Marca & $\begin{array}{c}\text { Capacidade } \\
\text { de frio }(\mathbf{k W})\end{array}$ & COP & $\begin{array}{c}\text { Investimento } \\
\text { (MUSD/equipamento) }\end{array}$ & $\begin{array}{c}\text { Custo O\&M } \\
\text { (USD/kWh) }\end{array}$ \\
\hline HuiN & $11,5-3.489$ & 0,7 & $9,96-170,0$ & 0,002 \\
\hline York & 1.200 & 1,0 & $166,6-168,0$ & 0,002 \\
\hline Sanyo & $30-525$ & 0,7 & - & 0,002 \\
\hline
\end{tabular}

Fonte: Autores, (2016).

Com os equipamentos que se relacionaram nos quadros acima, é possível construir a nova planta de fornecimento energético baseado na cogeração, estas tecnologias se combinam de acordo com as capacidades necessárias para as variáveis em questão. Na tabela 3 são apresentados os resultados do Cálculo de Pré-Viabilidade.

Tabela 3. Resultados Obtidos do Cálculo de Pré-Viabilidade

\begin{tabular}{|c|c|c|c|c|c|}
\hline \multirow[b]{2}{*}{ Tecnologia } & \multicolumn{3}{|c|}{ Alternativa 1} & \multicolumn{2}{|l|}{ Alternativa 2} \\
\hline & Caldeira & $\begin{array}{l}\text { Turbina de } \\
\text { Vapor }\end{array}$ & York & $\begin{array}{l}\text { Microturbi } \\
\text { na de Gás }\end{array}$ & HuiN \\
\hline Potencia Instalada kW & & 350 & 1.200 & 500 & 700 \\
\hline Potencia Necessária & & 387 & 1.196 & 500 & 688 \\
\hline Horas de Trabalho h/ano & 6.132 & 6.132 & 6.132 & 6.132 & 6.132 \\
\hline Custo de Investimento & 100.000 & 630.000 & 1680 & 450.000 & 170.000 \\
\hline Operação e Manutenção (USD/ kWh) & 0,02 & 0,0027 & 0,002 & 0,01 & 0,002 \\
\hline Quantidade (Unidades) & 1 & 1 & 1 & 1 & 1 \\
\hline Custo Total O\&M USD/ano & $42.924,00$ & $5.794,74$ & $\begin{array}{l}14 \\
667,74\end{array}$ & $30.660,00$ & $8.437,63$ \\
\hline Custo de Combustível (USD/ano) & \multicolumn{3}{|l|}{$1.155,90$} & \multicolumn{2}{|l|}{$1.192,98$} \\
\hline Custo de Investimento BCHP (USD) & \multicolumn{3}{|l|}{$731.680,00$} & \multicolumn{2}{|l|}{$620.000,00$} \\
\hline Custo Total de Operação & \multicolumn{3}{|l|}{$64.542,38$} & \multicolumn{2}{|l|}{$40.290,61$} \\
\hline Taxa Investimento $(\%)$ & \multicolumn{3}{|l|}{15} & \multicolumn{2}{|l|}{15} \\
\hline Vida Útil da Instalação (anos) & \multicolumn{3}{|l|}{20} & \multicolumn{2}{|l|}{20} \\
\hline Anual (USD/ano) & \multicolumn{3}{|l|}{$263.680,91$} & \multicolumn{2}{|l|}{$377.939,89$} \\
\hline Valor Atual Líquido (USD) & \multicolumn{3}{|l|}{$685.266,47$} & \multicolumn{2}{|l|}{$1.637 .911,11$} \\
\hline Taxa Interna de Retorno, TIR (\%) & \multicolumn{3}{|l|}{14} & \multicolumn{2}{|l|}{56} \\
\hline $\begin{array}{lll}\text { Período de Recuperação } & \text { do } \\
\text { Investimento (simples), (PRI) anos. } & \end{array}$ & \multicolumn{3}{|l|}{3,0} & \multicolumn{2}{|l|}{1,7} \\
\hline
\end{tabular}

Fonte: Autores, (2016).

A análise do quadro acima demonstra que a alternativa 2 é a de melhores resultados alcançados desde o ponto de vista econômico, pois tem os maiores valores do VAN e o TIR. O VAN é de 1,6 milhões de dólares por tanto é uma medida de ganhos que pode reportar esse projeto, sendo positivo o saldo entre os benefícios e gastos, em que a TIR é de $56 \%$, superior a $14 \%$ da alternativa 1

\section{IV.2.3 MELHORAS NO SERVIÇO QUE FORNECEM AS ALTERNATIVAS DE ABASTECIMENTO ENERGÉTICO PROPOSTA}

As alternativas propostas neste trabalho têm como objetivo principal, mudar o sistema de fornecimento energético atual do hospital, por um esquema de fornecimento muito mais eficiente, com o qual se poderão recuperar alguns serviços que atualmente se encontram fora de uso, aumentando assim a qualidade dos serviços prestados e as condições mínimas indispensáveis que requer uma instalação de saúde desta importância.

Com essas alternativas, poder-se-á dar solução aos seguintes problemas:

- Reabilitação ou substituição total do sistema de água quente para o asseio dos sanitários, o qual neste momento e desde alguns anos se encontra inoperante devido a saída do serviço dos aquecedores de água acionados por vapor.

- Permite a recuperação a carga total dos serviços de lavanderia e esterilização, também ambos em sérios problemas de funcionamento, os quais constituem um fator sensível no funcionamento adequado do hospital. 
- Se aumenta o número de locais climatizados e com ele o conforto nas salas do hospital.

Em todos os casos estas alternativas propostas no capitulo anterior, permitiram satisfazer eficientemente as demandas de energia que aumentariam sem duvida alguma, não somente a qualidade dos serviços prestados, como também o bem estar do pessoal clínico, os pacientes e seus acompanhantes em sua passagem por esta instituição.

\section{IV.3 IMPACTO AMBIENTAL}

Os riscos para a saúde quando se excedem as recomendações sobre qualidade do Ar da Organização Mundial da Saúde (OMS), variam muito e vão desde os sintomas de dificuldades respiratórias passando pelos efeitos hematopoiéticos e pelos efeitos cognitivos neurológicos até a carcinogêneos. Sendo difícil estimar a exposição real dos habitantes urbanos a contaminação do ar.

A partir dos anos 60 se começa a compreender realmente a necessidade de tomar medidas para contrariar os efeitos prejudiciais da contaminação do ar coincidindo com o auge da construção e montagem de industrias, as quais em sua imensa maioria foram executadas no ambiente ou dentro das principais cidades do país. As circunstâncias socioeconômicas do momento, unidas a falta de experiência enquanto a uma correto análise para a localização das industrias, somado ao feito de que numerosas industrias foram instaladas sem o sistema de depuração requerido, motivaram a certa diminuição da qualidade do ar em alguns assentamentos. Por ele muitas de nossas cidades se encontram contaminadas por cima dos limites permitidos.

Segundo um estudo realizado pela oficina nacional de normalização, de acordo com as normas que regem a concentração de contaminadores do ar e a NC TS 803: 2010 o nível de contaminação nas cidades tem seis possíveis níveis, estes são: critico, péssimo, mal, deficiente, aceitável e bom. O nível péssimo tem lugar em cidades que tem uma forte concentração industrial. $\mathrm{Se}$ adiciona a este grupo cem fogos, ainda quando as fontes contaminantes mais potentes, estão algo muito no centro da cidade.

Por outro lado, o nível de contaminação ruim o alcança, pela potência das suas fontes, os assentamentos urbanos que manifestam desenvolvimento industrial e por sua vez são capitais provinciais com maior densidade populacional. O nível de contaminação deficiente corresponde com as capitais província que tem um nível de desenvolvimento industrial inferior e uma população, relativamente baixa. Algumas apesar de não contar com uma população elevada, tem fontes potentes de contaminação (cimento e química respectivamente).

\section{IV.4 IMPACTO DAS ALTERNATIVAS DE FORNECIMENTO ENERGÉTICO SOBRE A QUALIDADE DO AR NA ÁREA HOSPITALAR.}

As alternativas de fornecimento energético propostas contribuem para a redução dos consumos globais de combustíveis a respeito do ano de 2010, entretanto, os consumos de combustíveis para o hospital aumentaram, porque é necessário analisar o impacto ambiental sobre a qualidade do ar destas propostas na área hospitalar, que se encontra tão contaminada.

As emissões estimadas para um ano em funcionamento são de aproximadamente 2.600 toneladas de $\mathrm{CO}_{2}$, uma tonelada de $\mathrm{CO}$, três toneladas de $\mathrm{NOx}$ e 121 toneladas de $\mathrm{SO}_{2}$ para a alternativa 2 .

$\mathrm{Na}$ tabela 4 se expõem os aumentos ou as reduções da alternativa 2 .

Tabela 4. Resumo das Emissões da Alternativa 2 com relação a 2010.

\begin{tabular}{c|c|c}
\hline \multirow{2}{*}{ Contaminantes } & \multicolumn{2}{|c}{ Reduções em relação a 2010 } \\
\cline { 2 - 3 } & Locais & Globais \\
\hline $\mathrm{CO}_{2}[\mathrm{t} / \mathrm{ano}]$ & 1706 & -3767 \\
\hline $\mathrm{CO}[\mathrm{t} / \mathrm{ano}]$ & 0,8 & $-0,3$ \\
\hline $\mathrm{NOx}[\mathrm{t} / \mathrm{ano}]$ & 0,8 & -15 \\
\hline $\mathrm{SO}_{2}[\mathrm{t} / \mathrm{ano}]$ & 79 & -174 \\
\hline \multicolumn{2}{|c}{ Fonte: Autores, (2016). }
\end{tabular}

Da análise do quadro anterior se pode dizer que existem incrementos locais de gases contaminantes, porém, as reduções globais são muito maiores. Os aumentos se estimam em 1787 toneladas de gases contaminantes (em conjunto), enquanto que as reduções globais totais se estimam em 3957 toneladas de gases contaminantes, muito superior aos aumentos.

\section{IV.5 ANALISES E DISCUSSÕES DOS RESULTADOS OBTIDOS}

As alternativas de fornecimento energético propostas contribuem com o aumento da eficiência energética do hospital, a diminuição do consumo total de combustível e dos custos totais de itens energéticos, assim como a redução global de emissões contaminantes, fatores de vital importância na seleção de um determinado projeto, como são apresentados os dados na tabela 5. 
Tabela 5. Aspectos Fundamentais de comparação entre as alternativas 1 e 2.

\begin{tabular}{l|c|c}
\hline Parâmetros & $\mathbf{1}$ & $\mathbf{2}$ \\
\hline Economia de Combustível [tcc/ano] & 210 & 936 \\
\hline Economia Energético (MUSD/ano] & 98 & 161 \\
\hline Custos de Investimento [MUSD] & 731,7 & 620 \\
\hline Custos Totais de O\&M [MUSD/ano] & 64,5 & 58,7 \\
\hline VAN [MUSD] & 685,3 & 1638 \\
\hline TIR [\%] & 14 & 56 \\
\hline PRI [anos] & 3,0 & 1,7 \\
\hline Emissões Locais Totais [t/ano] & 5684 & 2750 \\
\hline Emissões Globais Totais [t/ano] & 6059 & 2750 \\
\hline Fon & &
\end{tabular}

Fonte: Autores, (2016).

Na alternativa 2 é possível satisfazer em $100 \%$ a demanda

O resumo que aparece no quadro 10 dos principais resultados das alternativas estudadas nos capítulos deste trabalho constituem base fundamental da seleção de uma ou outra variante. Um balance destes resultados deixa muito claro que a alternativa 2 , é a mais idônea dentro dos dois pontos de vista, pois é a variante de maior economia de combustíveis, dos maiores índices econômicos monetários, e é a de menor custo de investimento, a de maior valor de VAN e de TIR (Taxa Interna de Retorno), assim como a de menor impacto ambiental, além do que o período de recuperação simples do investimento é menor.

A tecnologia usada para esta variante (2) selecionada seria uma microturbina de marca capstone de $500 \mathrm{~kW}_{\mathrm{e}}$ de potência de um sistema de refrigeração por absorção de água, bromuro de lítio de marca HuiN, com uma capacidade de frio de $700 \mathrm{~kW}_{\mathrm{t}}(195$ TR). Este investimento custaria uns 260 mil dólares e permitiria um retorno do investimento em aproximadamente uns dois anos, a qual se pagaria fundamentalmente pela economia líquida durante seu processo de operação ao substituir o sistema atual de fornecimento energético.

\section{CONCLUSÕES}

Para efeitos de comparação, análise e tomada de decisão acerca de qual alternativa adotar para a estrutura do hospital alpha chegou-se as seguintes conclusões baseadas na aplicação do modelo matemático escolhido, como também, a estrutura do modelo definido conforme segue:

Na alternativa 1, percebeu-se que é possível satisfazer totalmente a demanda de calor para o aquecimento de água, vapor para outros usos e vapor para a planta de refrigeração por absorção de umas 340 TR. Também é possível satisfazer em 89\% a demanda de energia elétrica a qual compreende $106 \mathrm{kWe}$ para climatização local e $281 \mathrm{kWe}$ para outros consumos, além do mais se pode economizar 210 tcc/ano que equivalem a $10 \%$ do combustível total empregado, representando uma economia de 131 MUSD/ano por este conceito. Esta alternativa permite economia líquida total de $98 \mathrm{MUSD} / \mathrm{ano}$. de eletricidade, ou seja, uns $493 \mathrm{kWe}$ que compreendem $212 \mathrm{kWe}$ para climatização local e $281 \mathrm{kWe}$ para outros consumos, esta alternativa permite além disso tudo satisfazer em sua totalidade a demanda de calor para alimentar uma planta de refrigeração por absorção de umas 195 TR, também permite satisfazer a demanda de água quente $(214 \mathrm{kWt})$, e em $94 \%$ da demanda de vapor para outros usos, o resto do vapor para outros usos se pode gerar nas caldeiras existentes. Nesta alternativa é possível economizar em $43 \%$ o uso e consumo do combustível, o que representa uma economia de 936 tcc/ano, sendo a economia líquida mínima de 161 MUSD/ano. Esta variável desde o ponto de vista energético e monetário é a que representa melhores resultados, pois é a que mais traz meios de economia no uso do sistema de cogeração. É possível dizer que a economia de energia pode chegar a ser maior se os cálculos se realizarem tomando por base o preço do GLP importado e este ser um combustível que se pode obter diretamente em nosso país, porque sempre será mais barato produzi-lo do que compra-lo no mercado, aliás esta cifra pode ser afetada pelo preço do combustível como se havia dito anteriormente, e os benefícios em alguns meses ou anos podem chegar a serem maiores se se manterem a turbina trabalhando a plena capacidade buscando manter elevada eficiência da mesma e permitindo eventualmente exportar ao SEN a energia elétrica que sobra devido ao balance da demanda. E isto também pode suceder de forma contrária, porém sem nenhuma dúvida esta é uma boa alternativa para se pôr em prática.

As alternativas propostas neste estudo têm como objetivo fundamental, transformar o sistema de fornecimento energético atual do hospital Alpha, local em que foi desenvolvida toda a pesquisa, análise de dados, consumos, equipamentos e layout, buscando um esquema de fornecimento muito mais eficiente, com o qual se possam recuperar alguns dos serviços prestados e as condições mínimas indispensáveis que requerem uma organização de saúde desta importância.

Com estas alternativas pode-se chegar à conclusão de que atingirá a solução dos seguintes problemas: 
Reabilitação ou substituição total do sistema de água quente para o banho dos pacientes, acompanhantes, para o pessoal dos serviços gerais, assim como para outros usos sanitários, o qual nestes momentos e há alguns anos se encontram inoperantes, devido a saída de serviço dos aquecedores de água acionados por vapor.

Permite a recuperação da carga total dos serviços de lavanderia e esterilização, também ambos, em sérios problemas de funcionamento adequado para o hospital.

Se aumenta o número de locais climatizados e com eles o conforto nas salas do hospital, melhorando a qualidade dos serviços prestados.

Em todos os casos estas alternativas propostas no capítulo anterior, permitiram satisfazer eficientemente diversas demandas de energia que aumentariam sem dúvida alguma, não somente a qualidade dos serviços prestados, como também o bem estar do pessoal clínico, dos pacientes e seus acompanhantes em sua passagem pela instituição.

A alternativa 2 selecionada é a de melhores resultados econômicos e financeiros pois o VAN é de 1,6 milhões de dólares e a TIR de $56 \%$ após o período citado nessas analises.

\section{REFERÊNCIAS}

[1] Veiga, T. P. (2007). "<Projeto de Planta de Cogeração para um Hospital>." USP.

[2] Alexis, G. K. and P. Liakos (2013). "A case study of a cogeneration system for a hospital in Greece. Economic and environmental impacts." Applied Thermal Engineering 54(2): 488-496.

[3] Ziher, D. and A. Poredos (2006). "Economics of a trigeneration system in a hospital." Applied Thermal Engineering 26(7): 680687.

[4] Carvalho, M., M. A. Lozano and L. M. Serra (2012). "Multicriteria synthesis of trigeneration systems considering economic and environmental aspects." Applied Energy 91(1): 245-254.

[5]Sales, L. d. S. (2008). "Viabilidade Técnico Econômica Cogeração.pdf." Universidade Federal de Itajubá (UNIFEI).Tese de Doutorado.

[6] Lizarraga, J. M. S. (1999). "Cogeneracíon: Aspectos Termodinâmicos, Tecnológicos y Económicos." Bilbao: Ed. Universidad del País Vasco.

[7] KoronakiS, I. (2004). "Co-generation, in Air ConditioningEnergy Consumption and Environmental Quality." Encyclopedia of Life Support Systems (EOLSS), Developed under the Auspices of the UNESCO. Oxford, UK: Eolss Publishers.

[8] Pagliarini, G., C. Corradi and S. Rainieri (2012). "Hospital CHCP system optimization assisted by TRNSYS building energy simulation tool." Applied Thermal Engineering 44: 150158.
[9] Gimelli, A. and M. Muccillo (2013). "Optimization criteria for cogeneration systems: Multi-objective approach and application in an hospital facility." Applied Energy 104: 910923.

[10] Gil, A. C. (2000). "Metodologia do Conhecimento Científico." ATLAS.

[11] Gonçalves, C. A. M., Anthero de Moraes. (2010). "Projetos e Relatórios de Pesquisa em Administração." São Paulo: Atlas. 